\title{
Calidad de vida de los mayores que viven institucionalizados en residencias para mayores: Un análisis cuantitativo
}

\author{
Esther Acevedo Alcaraz \\ Universidad de Murcia (España)
}

Las residencias para mayores parecen "instituciones cerradas" o "microcosmos independientes" centrando el interés de los medios de comunicación sólo en las deficiencias de las residencias para mayores. Objetivos: comparar la calidad de vida, capacidad funcional, el grado dependencia y los síntomas depresivos de las personas mayores institucionalizadas en residencias comparándolas con los mayores que residen junto a sus familiares. Material y método: se han analizado la calidad de vida y las alteraciones físicas y psicológicas de 400 personas mayores de 60 años de edad, ingresados en residencias de mayores o que viven junto con sus familiares, mediante la realización de un cuestionario que recoge tres índices diferentes (Barthel Index, escala de Yesavage y cuestionario WHOQOL-OLD) para evaluar el grado de dependencia de las personas. Resultados: se ha determinado una reducción significativa de la calidad de vida de las personas mayores que viven en residencias $(p<0.001)$, respecto a los mayores que conviven habitualmente con sus familiares. Nuestros resultados muestran que el grado de dependencia física y/o psicológica de nuestros mayores es el factor determinante para su ingreso en una residencia para mayores en nuestra comunidad $(p>0.001)$. Conclusión: el modelo actual de residencias requiere un cambio que permita dar respuestas a las necesidades reales de nuestros mayores institucionalizados.

Palabras clave: Personas mayores, residencias para mayores, cuidados de enfermería, envejecimiento, dependencia.

Quality of life of institutionalized elderly people in nursing homes: a quantitative analysis. Nursing homes have the characteristic of being "complete institutions" or "enclosed microcosms" and the quality of life (QoL) in late adulthood is generally perceived as a multidimensional construct. Over recent years, media reports have focused on the deficiencies in nursing home residences, and a subsequent increase in the demand for instruments capable of measuring this construct has arisen. Objective: To compare the quantitative functional mobility, level of independence, depressive symptoms and QoL of nursing home residents compared with home care recipients. Methods: have analyzed the quality of life and physical and psychological disorders 400 people over 60 years old, admitted to nursing homes or living with their families through the implementation of a questionnaire covering three different indices (Barthel Index, Yesavage and WHOQOL-OLD) to assess the degree of dependence of people. Results: has resulted in a significant reduction in quality of life of older people living in nursing homes $(p<0.001)$ compared to older usually live with their families. Our results show that the degree of physical and / or psychological dependence on our largest is the determinant for entry into a nursing home in our community $(p>0.001)$ factor. Conclusions: the current model residences require a change that allows responding to the real needs of our elderly institutionalized.

Keywords: Elderly, nursing home, aged, nursing care, dependency.

Correspondencia: Esther Acevedo. Departamento de Medicina Física y Radiología. Facultad de Medicina. Instituto Universitario de Investigación en Envejecimiento. Universidad de Murcia. Campus Espinardo. C.P.: 30100. Murcia (España). E-mail: esther.acevedo@um.es 
La investigación gerontológica ha dedicado siempre un esfuerzo considerable para la identificación de las características que pueden mostrar las variaciones en la calidad de vida durante el envejecimiento. Sin embargo, hay pocos estudios que se centren específicamente en las personas que pasan sus últimos años en una residencia de ancianos y aún menor el número de estudios que comparan sus resultados con los mayores que residen en sus casas junto a sus familiares (Aberg et al., 2005.; Lang et al., 2007; Pieper y Vaarama, 2008; Cooney et al., 2009.; Schenk et al., 2013). Posiblemente, una de las razones de esta falta de investigación o interés sea debido a las características particulares de las residencias para mayores que prácticamente se han comportado como una institución aislada e independiente e incluso como un microcosmos cerrado (Koch-Straube et al., 2003; Cooney et al., 2009; Schenk et al., 2013), siendo difícil acceder a las personas mayores institucionalizadas y detectar sus características sociales y sanitarias.

Hoy día se considera que la calidad de vida de las personas mayores debe ser percibida como situación con estructura multidimensional (Lawton 1991; Schenk et al., 2013). Por lo menos, se hace una distinción entre los aspectos "objetivos" y "subjetivos" en la evaluación de sus características. Los aspectos objetivos de la calidad de vida se refieren principalmente a la evaluación de las condiciones de vida (la nutrición, movilidad, vivienda) y algunos otros los estándares definidos por los expertos; por lo general se evalúa a través de parámetros externos. Los aspectos subjetivos de la calidad de vida se refieren a las características de la experiencia de vida (la satisfacción en las condiciones afectivas, el sentido del bienestar, etc.) y es, por definición, evaluados en relación con las normas individuales que se valora directamente por la propia persona (Damián et al., 2013.; Lucas y Dienner, 2008; Fernández-Ballesteros, 2011).

En los últimos años, los medios de comunicación se suelen centrar en los déficits y escándalos relacionados con el cuidado de ancianos en estas residencias para mayores. Además, han despertado un considerable interés público sobre las condiciones de calidad de vida de las personas mayores institucionalizadas en residencias (OECD, 2005; Schenk et al., 2013). Como consecuencia, se ha suscitado la demanda de instrumentos capaces de medir la calidad de vida en estas situaciones (Kelle y cols, 2008; Schenk et al., 2013).

Nuestro estudio pretende cuantificar algunos aspectos tanto objetivos como subjetivos de la calidad de vida de nuestros mayores institucionalizados en residencias, comparándola con la obtenida en una población similar que permanecen viviendo en compañía de sus propias familias; en un intento de valorar las necesidades reales que nos permitan elaborar formas de intervención social que mejoren los problemas de integración de estos mayores dentro y fuera de estas instituciones. 


\section{MÉTODO}

Entre enero de 2011 y enero de 2014 se han entrevistado 400 personas mayores de 60 años con dos características diferentes: a) personas mayores institucionalizadas en residencias de la Comunidad Autónoma de la Región de Murcia (300 personas entrevistadas) y b) personas mayores de similares características socioculturales que residen habitualmente con su familia (100 personas entrevistadas); analizando las capacidades físicas, las alteraciones psicológicas, los problemas de integración social y la calidad de vida que presentan. En todos los casos se ha establecido una relación directa entre las poblaciones encuestadas de ambos grupos (residentes y en familias) con su lugar de residencia, sexo y edad.

Los mayores esta institucionalizados en cinco residencias para mayores ubicadas en la Comunidad Autónoma de la Región de Murcia, de carácter privado que ofertan numerosas plazas concertadas con los organismos públicos cuyos directores han permitido el estudio en sus centros obteniéndose por escrito el consentimiento informado de cada participante. Los entrevistados eran de ambos sexos con un predominio del sexo femenino (65\% mujeres, 35\% hombres) y edades comprendidas entre 60 y 102 años (edad media=77.8 años). Sus características sociodemográficas se muestran en la tabla 1.

Tabla 1. Características demográficas y sociales de la población de mayores que institucionalizadas en residencias y las que viven junto a sus familias $(* p<0.001$ respecto a los mayores institucionalizados en residencias para mayores)

\begin{tabular}{|c|c|c|c|}
\hline & & Residencias $(n=350)(\%)$ & Familia $(n=150)(\%)$ \\
\hline \multirow{2}{*}{ Género: } & Mujer & 70 & 60 \\
\hline & Hombre & 30 & 40 \\
\hline \multirow{5}{*}{$\begin{array}{l}\text { Rango de edad } \\
\text { (años): }\end{array}$} & $60-69$ & 8.9 & 10.7 \\
\hline & $70-79$ & 30.7 & 40 \\
\hline & $80-89$ & 42.6 & 42.7 \\
\hline & $90-99$ & 15.8 & 6.6 \\
\hline & $\geq 100$ & 2 & 0 \\
\hline \multirow{3}{*}{ Número de hijos: } & 1 & 31.7 & $9.3 *$ \\
\hline & 2 & 17.8 & 6.7 \\
\hline & $\geq 3$ & 28.7 & $60^{*}$ \\
\hline \multirow{4}{*}{ Nivel de estudios: } & Analfabeto & 24.8 & 23.4 \\
\hline & Primarios & 40.6 & 34.3 \\
\hline & Secundarios & 30.6 & 35.7 \\
\hline & Universidad & 4 & 6.7 \\
\hline \multirow{3}{*}{ Estado Civil: } & Soltero/a & 17.8 & $5.3^{*}$ \\
\hline & Casado/Viudo & 66.3 & 54.7 \\
\hline & Divorciado & 57.4 & $40^{*}$ \\
\hline \multirow{6}{*}{$\begin{array}{l}\text { Nivel económico } \\
(€ / \text { mes): }\end{array}$} & $\leq 300$ & 6 & 0 \\
\hline & $301-600$ & 42.7 & 33 \\
\hline & $601-900$ & 35.8 & 39.7 \\
\hline & $901-1.200$ & 7.9 & 11.4 \\
\hline & $1.201-1.800$ & 5 & 14.7 \\
\hline & $\geq 1.801$ & 1 & 1.3 \\
\hline
\end{tabular}


La recogida de datos se realizó a través de una entrevista personalizada de los autores, mediante la realización un cuestionario previamente establecido que comprende 77 preguntas que recogen los índices y cuestionarios estandarizados que se pretenden evaluar. Se han estudiado diferentes variables.

Variables sociodemográficas: sexo, edad, nivel educativo, estado civil, asistencia social, número de hijos, pensiones.

La calidad de vida (QoL): se ha utilizado el cuestionario de edad avanzada de la Organización Mundial de la Salud Calidad de Vida de los Adultos Mayores (WHOQOL-OLD). Consta de 24 ítems que describen seis aspectos diferentes (habilidades sensoriales; autonomía; actividades pasadas, presentes y futuras, participación social, la muerte y la intimidad). La puntuación total es la suma de las puntuaciones individuales para cada una de las seis secciones, siendo el total de 100 (QoL General), en donde los valores más altos indican una mejor calidad de vida (WHOQOL, 1998).

Estado física o funcional: hemos utilizado el índice de Barthel (Mahoney y Barthel, 1965), que valora el grado de dependencia en las Actividades Básicas de la Vida Diaria (ABVD). Dicho índice evalúa el grado de dependencia para la realización de las siguientes actividades básicas de la vida diaria: comer, ir al baño, la higiene personal, bañarse/ducharse, vestirse/desvestirse, caminar, usar las escaleras, y la continencia urinaria/fecal. Los residentes fueron evaluados de acuerdo a las características del cuestionario: independientes (100 puntos), dependencia leve (91-99 puntos), dependencia moderada (21-90) y dependencia severa (0-20 puntos).

Síntomas depresivos: se evalúan mediante la versión corta de la escala de depresión geriátrica de Yesavage para determinar los síntomas depresivos según el criterio establecido para la evaluación en diferentes niveles (Yesavage et al., 1982): síntomas depresivos (siendo 0-5, 6-9 y 10-15 puntos, cuyas áreas de distribución indican síntomas de depresión (sin depresión, depresión leve y depresión establecida, respectivamente).

El análisis estadístico ha consistido en el estudio del grado de dependencia y correlación entre variables mediante análisis de varianza complementado por el contraste de medias. Las variables cuantitativas se compararon mediante análisis de regresión y correlación lineal. Los resultados se consideraron estadísticamente significativas a un valor de $\mathrm{p}$ de menos de $0.01(p<0.01)$.

Nuestros resultados muestran que el perfil de la persona mayor que vive en una residencia para mayores es una mujer entre 70 y 89 años, que no tiene pareja (viuda o divorciada), con algún hijo, estudios básicos, ingresos inferiores a $600 € / m e s$, que vive permanente en la residencia compartiendo habitación con otra persona y presenta una significativa dependencia física/psicológica. 
La puntuación obtenida mediante el cuestionario de Calidad de Vida en las personas mayores de la OMS (WHOQOL-OLD) es significativamente inferior en los mayores institucionalizados en residencias ( $33 \pm 14$ puntos) que la determinada en los mayores que habitan con sus familias $(54,7 \pm 12,1)$ apreciándose diferencias estadísticamente significativas $(p<0.001)$, determinándose un 39,7\% de reducción en su calidad de vida y mostrando una disminución significativa de la calidad de vida de los mayores que viven en residencias respecto a los mayores que conviven habitualmente con sus familias.

Esta disminución global de la calidad de vida se produce fundamentalmente en las diferencias determinadas en la disminución de su autonomía personal $(p<0.001)$ y en los aspectos de mantenimiento de su propia intimidad $(p<0.001)$, apoyados también por la disminución significativa de su participación social $(p<0.01)$. Sin embargo la percepción del mantenimiento de sus habilidades sensoriales y percepciones de sus actividades pasadas, presentes y actitud frente a la muerte son similares en ambas poblaciones de mayores sin diferencias significativas entre los que viven en residencias o viven con sus familias (Figura 1).

Figura 1. Calidad de vida (cuestionario WHOQOL-OLD) en ancianos institucionalizados en residencias en comparación con los que viven en su domicilio. Con un error estándar de la media $\pm\left({ }^{*} p<0.001\right.$ frente a personas mayores que viven en su hogar)

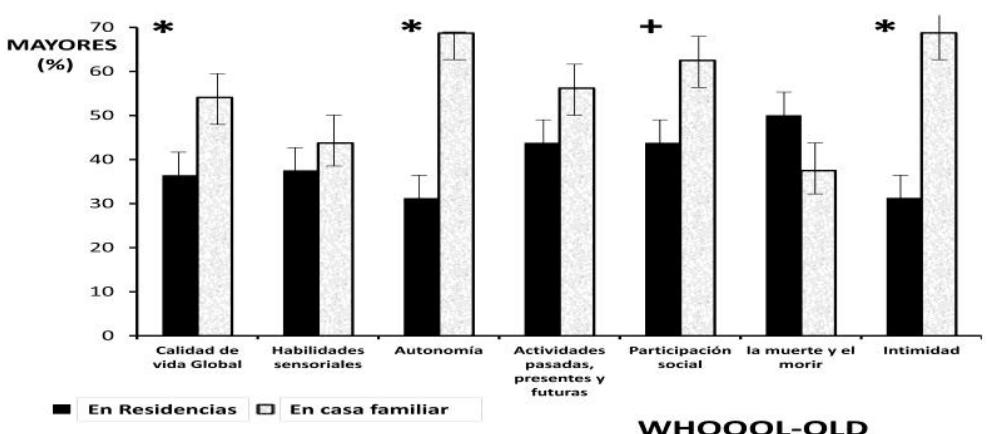

Mediante el índice de Barthel, que valora la capacidad para realizar las actividades básicas de la vida diaria de forma autónoma, nuestros resultados muestran un grado severo o total de dependencia en el $47.2 \%$ de los pacientes que viven en residencias; mientras que sólo el 15.2\% de los residentes son completamente autónomos, apreciándose diferencias significativas respecto a los mayores que viven con sus familias $(p<0.001)$ y que expresarían el mayor grado de dependencia para estas actividades cotidianas de la vida diaria de los mayores institucionalizados en residencias (Figura 2). 
Figura 2. Índice de Barthel. Grado de dependencia para las actividades básicas de la vida diaria de las personas mayores institucionalizadas en residencia para mayores respecto a los que viven con su familia. Error estándar de la media $\pm(* p<0.001)$

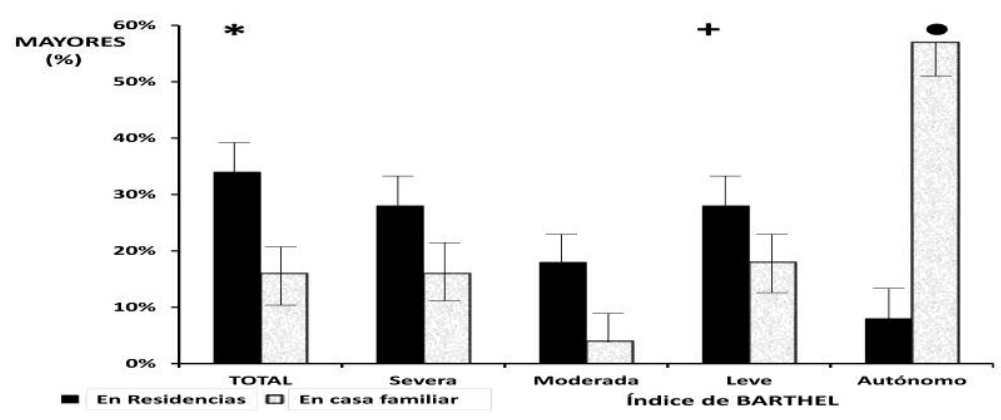

Las personas mayores que viven de forma permanente en una residencia para mayores presenta además, las diferencias estadísticamente significativas en comparación con los que viven en el país: el $79 \%$ no son capaces de subir escaleras $(p<0.001)$, el $67 \%$ va vagando $(p<0.001)$, e incluso el $64 \%$ no pueden caminar o moverse de forma independiente $(p<0.001)$. Además, el $60 \%$ necesita ayuda para vestirse o con la higiene personal, e incluso para orinar $(p<0.001)$ y el $47 \%$ necesita ayuda para comer $(p<0.01)$.

Mediante la escala de depresión geriátrica de Yesavage que evalúa los trastornos psicológicos y la prevalencia de los síntomas depresivos en las personas mayores, hemos determinado que el $47 \%$ de la población institucionalizada en residencias un grado leve (32\%) o establecido (15\%) de síntomas depresivos; no apreciando diferencias significativas en comparación con las personas que viven con su familia. Sin embargo, nuestros resultados muestran un menor número de características "normales" en los ancianos que viven en residencias en comparación con los que viven con sus familias $(p<0.01)$ (Figura 3$)$.

Figura 3. Escala de Yesavage: grado de los síntomas depresivos en los ancianos institucionalizados que viven en residencias en comparación con los que viven con su familia. Error media estándar $\pm(* p<0.001)$ frente a personas mayores que viven en su casa)

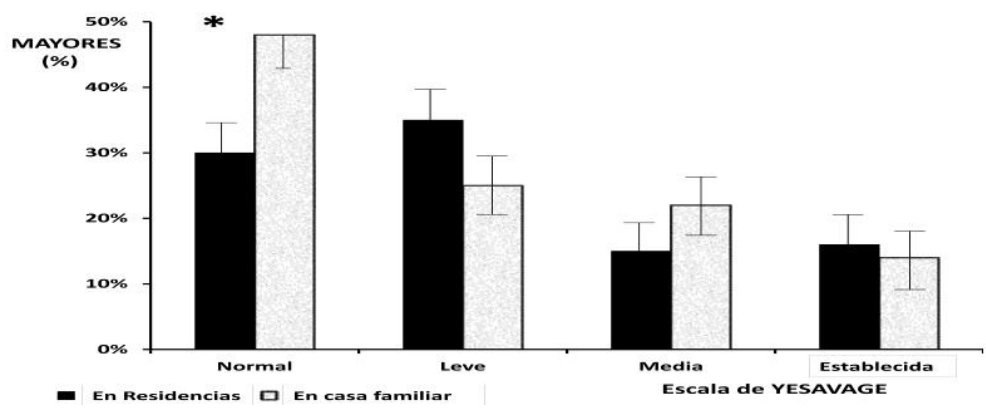




\section{DISCUSIÓN}

Diferentes autores han escrito una mayor movilidad funcional, mejor nivel de independencia, menor número de síntomas depresivos e incluso mejor calidad de vida de los mayores institucionalizados en residencias para mayores que en los mayores que residen habitualmente en domicilios junto con sus familiares (Tada et al., 1999; Kerem et al., 2001; Suhin y Yalem , 2003; Ozer , 2004; Chung , 2008; Karakaya et al., 2009; Yumin et al., 2011). Con frecuencia, algunos estudios describen que las personas mayores que viven en residencias tienen mejor salud y requieren menos cuidados de enfermería que los que viven en casa junto con sus familias (Erdil et al., 2006; Damian et al., 2013; Karakaya et al., 2009). Incluso se ha descrito que los mayores en residencias son más independientes en su cuidado personal y menos sedentarios que los ancianos que viven en casa con sus familiares (Kerem et al., 2001; Karakaya et al., 2009). Por el contrario, nuestros resultados muestran que los ancianos que viven en residencias tienen un mayor grado de dependencia física, numerosas necesidades de atención física, mayor deterioro cognitivo, síntomas depresivos más frecuentes y unas relaciones sociales y familiares menores que los mayores que residen con sus familias; siendo incluso el factor determinante para la institucionalización del mayor en una residencia de forma permanente (Acevedo et al., 2011; Acevedo et al., 2014).

Mientras que nuestros resultados de la calidad de vida de los mayores que viven con su familia son similares a los descritos por otros autores (Urzúa et al., 2011; Vitorino et al., 2012), la calidad de vida de las personas mayores que viven en residencias es significativamente menor que la de los que viven en casa con sus familias. En este sentido, algunos autores han descrito que los mayores que viven en un entorno familiar tiene un sentido más alto de satisfacción con su propia vida en comparación con los que viven en residencias (Ozer, 2004). Incluso, se ha descrito que la calidad de vida de personas mayores cuando comienzan a vivir en una residencia percibe un deterioro en su calidad de vida sólo 6 meses tras su incorporación a una residencia de mayores (Scocco et al., 2006). Los sentimientos de soledad y marginación se encuentran entre las razones de ese descenso en la calidad de vida percibida (Scocco et al., 2006).

Se ha descrito que la calidad de vida relacionada con la atención institucional en las residencias para mayores depende de diferentes factores destacando las relaciones interpersonales (por ejemplo, "escuchar si el mayor plantea preocupaciones"), y también en la "dimensión de contactos sociales (Schenk et al., 2013). El apoyo psicológico, fomentar la participación en actividades de grupo y la interacción social beneficia la percepción individual de la calidad de vida y reduce la frecuencia de síntomas depresivos. Las actividades externas al aire libre pueden mejorar el bienestar al proporcionar un sentido de autonomía, y puede mejorar la eficiencia fisiológica a través 
de ejercicio apoyando la integración social mediante la vinculación de las personas activas como amigos y familiares un contexto estructurado (Karakaya et al., 2009).

En nuestro estudio la percepción de pérdida en la calidad de vida en las residencias de mayores se encuentra especialmente en los aspectos de autonomía personal, relaciones y participación social así como en el mantenimiento de la intimidad. Los mayores que viven en una residencia realizan actividades fundamentalmente dentro de las propias residencias, limitando las conversaciones con amigos y familiares e incrementando las horas de televisión (Csapo et al., 2009; Yumin et al., 2011).

En España, sólo hay un único modelo generalizado de residencia de mayores a diferencia de los países europeos de nuestro entorno, que no se adapta a las necesidades reales que hemos detectado en nuestros mayores institucionalizados en residencias, poniendo de manifiesto que deberíamos abordar dicho modelo para dar una respuesta a los problemas reales de nuestros mayores institucionalizados en residencias para mayores.

\section{CONCLUSIÓN}

Las necesidades de la población de mayores institucionalizada en residencias para mayores han cambiado significativamente en los últimos años, y nos encontramos arrastrando un modelo único de residencia de ancianos obsoleto e inadecuado para las necesidades actuales de nuestros mayores.

\section{REFERENCIAS}

Aberg, A.C., Sidenvall, B., Hepworth, M., O’Reilly, K. y Lithell, H. (2005). On loss of activity and independence, adaptation improves life satisfaction in old age: A qualitative study of patients' perceptions. Quality of Life Research, 14, 1111-1125.

Acevedo, E., Alcaraz, M., Benito, J. y Fombuena, J. (2012). Diferencias entre las personas mayores alojadas en residencias y las que conviven con sus familiares en la comunidad autónoma de la Región de Murcia. Revista de Trabajo Social y Salud, 73, 301-310.

Acevedo, E., Alcaraz, M., Benito, J., Muir, B.R. y Navalón, C. (2014). Situación de nuestros mayores institucionalizados en residencias y necesidades para su integración social. Azarbe Revista Internacional de Trabajo Social y Bienestar, 3, 279- 282.

Chung, S. (2008). Residential status and depression among Korean elderly people: A comparison between residents of nursing home and those based in the community. Health Soc Care Community, 16, 370-377.

Cooney, A., Murphy, K. y O'Shea, E. (2009). Resident perspectives of the determinants of quality of life in residential care in Ireland. Journal of Advanced Nursing, 65, 1029-1038.

Csapo, R., Gormasz, C. y Baron, R. (2009). Functional performance in community-dwelling and institutionalized elderly women. Wien Klin Wochenschr, 121, 383-390.

Damian, J., Pedro-Cuesta, J., Almazán, J., Comín-Comín, M., Quintanilla, M.A. y Lobo, A. (2013). Depressive symptoms and associated factors in an older Spanish population positively screened for disability. Geriatric Psychiatric, 28, 744-55. 
Erdil, F., Celik, S.S., Elik, S. y Baybuga, M.S. (2006). Aging in Turkey. Malta: International Institute on Ageing, United Nations (INIA).

Fernández-Ballesteros, R. (2011). Quality of life in Old Age: Problematic Issues. Applied Research Quality Life, 6, 21-40.

Karakaya, M.G., Sevil, C., Bilgin, U., Ekici, G., Köse, N. y Otman, A.S. (2009). Functional Mobility, Depressive Symptoms, Level of Independence, and Quality of Life of the Elderly Living at Home and in the Nursing Home. Journal of the American Medical Directors Association, 10, 662-666.

Kelle, U., Niggemann, C. y Metje, B. (2008). Datenerhebung in totalen Institutionen als Forschungsgegenstand einer kritischen gerontologischen Sozialforschung. In: Amann, A.; Kolland, F. (Hrsg.): Das erzwungene Paradies des Alters? Fragen an eine kritische Gerontologie. Wiesbaden: VS Verlag für Sozialwissenschaften, S. 163-193.

Kerem, M., Meric, A., Kırd1, N. y Cavlak, U. (2001). Evaluation of elderly living at home and rest house. Turkish Journal of Geriatrics, 4, 106-112.

Koch-Straube, U. (1999). Immigrants in geriatric institutional care. Pflege, 5, 289-294.

Lang, G., Loger, B. y Amman, A. (2007). Well-being in the nursing home: A methodological approach towards the quality of life. Journal for Public Health, 15, 109-120.

Lawton, M.P. (1991). A multidimensional view of quality of life in frail elders. In: J. E. Birren, J. E. Lubben, J.C. Rowe, y D.E. Deutchmann (Eds.), The concept and measurement of quality of life elderly pp. 3-27. San Diego: Academic Press.

Lucas, R. y Diener, E. (2008). Subjective well-being. En M. Lewis, J.M. Haviland-Jones y L. Feldmann (Eds.), Handbook of emotions (pp. 471-485). New York: Guilford Press.

Mahoney, F.I. y Barthel, D.W. (1965). Functional evaluation: the Barthel Index. Maryland State Medical Journal, 14, 61-65.

Organisation for Economic Co-Operation and Development (OECD) (2005). Ensuring quality long-term care for older people. Policy Brief. Available from: http://www.oecd.org/dataoecd/53/4/34585571.pdf.

Özer, M. (2004). A study on the life satisfaction of elderly individuals living in family environment and nursing homes. Turkish Journal of Geriatrics, 7, 33-36.

Pieper, R. y Vaarama, M. (2008). The concept of care-related quality of life. En M. Vaarama, R. Pieper y A. Sixsmith (Eds.), Care-related quality of life in old age: Concepts, models and empirical findings (pp. 65-101). Springer New York.

Schenk, L., Meyer, R., Behr, A., Kuhlmey, A. y Holzhausen, M. (2013). Quality of life in nursing homes: results of a qualitative resident survey. Quality of Life Research, 2929-38.

Scocco, P., Rapattoni, M. y Fantoni, G. (2006). Nursing home institutionalization: A source of eustress or distress for the elderly? Int J Geriatr Psychiatry, 21, 281-287.

Suahin, E.M. y Yalem, B.M. (2003). Comparing the incidences of depression at the elderly living in nursing home or at their own homes. Turkish Journal of Geriatrics, 6, 10-13.

Tada, T., Yamamoto, S. y Morimoto, T. (1999). Characteristics of the daily lives of elderly women at various facilities for the elderly. Nurs Health Sci, 1, 13-18.

The World Health Organization Quality of Life Assessment (WHOQOL). Development and general psychometric properties. (1998). Soc Sci Med, 46, 1569-1585.

Urzúa, A., Bravo, M., Ogalde, M. y Vargas, C. (2011). Quality of life of older people living in Antofagasta, Chile. Rev Med Chile, 139, 1006-1014 (in Spanish).

Vitorino, L.M., Paskulin, L.M. y Viana, L.A. (2012). Quality of life among older adults resident in long-stay care facilities. Rev Lat Enfermagem, 20, 1186-1195.

Yesavage, J.A, Brink, T.L., Rose, T.L., Lum, O., Huang, V., Adey, M. y Leirer, V.O. (1982). Development and validation of a geriatric depression screening scale: A preliminary report. Journal of Psychiatric Research, 17, 37-49. 
Yümin, E.T., Simsek, T.T., Sertel, M., Özturk, A. y Yümin, M. (2011). The effect of functional mobility and balance on health-related quality of life (HRQoL) among elderly people living at home and those living in nursing home. Archives of Gerontoly and Geriatrics, $52,180-184$.

Recibido: 15 de abril de 2014 Recepción Modificaciones: 26 de mayo de 2014 Aceptado: 13 de agosto de 2014 